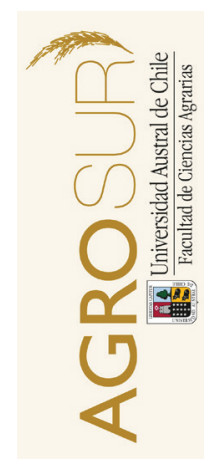

\title{
Comparación florística y alteración antrópica de tres turberas esfagnosas en la Región de Aisén, Chile: buscando indicadores de perturbación por manejo
}

\author{
Floristic comparison and anthropic alteration of three sphagnum bogs \\ in the Aisén Region, Chile: looking for management disturbance indicators \\ San Martín, C. ${ }^{a *}$, Montenegro, D. ${ }^{b}$, Vidal, O. ${ }^{c}$, Álvarez, I. ${ }^{a}$, Solís. J.L. ${ }^{a}$, Ramírez, C. ${ }^{d}$ \\ ${ }^{a}$ Instituto de Ciencias de la Tierra, Facultad de Ciencias, Universidad Austral de Chile, Casilla 567, \\ Valdivia, Chile. \\ ${ }^{b}$ Teniente Merino $\mathrm{N}^{\circ} 441$, Ingeniero Ejecución Agrícola, Consultora, Cochrane, Chile. \\ ${ }^{c}$ Universidad de Magallanes, Instituto de la Patagonia, Universidad de Magallanes. Avenida Bulnes 01855, \\ Punta Arenas, Chile. \\ ${ }^{d}$ Departamento de Ecología, Facultad de Ciencias Biológicas, Pontificia Universidad Católica de Chile. \\ Avenida Libertador Bernardo O’Higgins 240, Santiago, Chile.
}

\begin{tabular}{l} 
A R T I C L E I N F O \\
\hline Article history: \\
Received: 10.05.2018 \\
Accepted: 21.06.2018 \\
\hline Keywords: \\
Chilean Patagonia \\
Peat bogs vegetation \\
Plant sociology \\
Ordination \\
Classification \\
\hline Original Research Article, \\
Plant Science \\
\hline *Corresponding author: \\
Cristina San Martín \\
E-mail address: \\
csanmart@uach.cl
\end{tabular}

A R T I C LE INF O

A B S T R A C T

The flora of three Sphagnum bogs belonging to the Sphagnetum magellanicii Oberdorfer plant association located in the commune of Tortel (Aisén, Chile) was compared using analysis to identify plants indicators of their differences. We worked on the field by means of plant sociological methods and with classification and ordination multivariate statistical methods with an initial table with 30 vegetation samples taking 10 in each of the three study sites. Most plant species are native only one is introduced, Festuca rubra L. of European origin and biological spectrum of the flora was typical of extreme conditions. With traditional phytosociological methodology floristic differences between the vegetation samples of the three bogs and in statistical arrangements with few variations were found. With the ordination of the species were able to identify as important variations in humidity and minor in the accumulation of organic matter in the soil. Carex kingii R.Br. and Tetroncium magellanicum Willd. appear as moisture indicators while Nanodea muscosa Banks, Juncus scheuchzerioides Gaudich. and Festuca rubra of drought. Also Marssippospermum grandiflorum L.f. and Nothofagus antarctica G. Forst. were found occupying intermediate positions. It is concluded that the sites have different soil moisture conditions probably by anthropogenic drainage, the soil moisture regulating the degradation of organic matter. The site with the highest alteration was presented as more favorable for the operation of Sphagnum magellanicum Brid.

\section{RESUMEN}

Se comparó la flora de tres turberas esfagnosas, pertenecientes a la asociación Sphagnetum magellanicii Oberdorfer ubicadas en la comuna de Tortel (Aisén, Chile), utilizando análisis que permitan identificar plantas indicadoras representativas de sus diferencias. Se trabajó con metodología fitosociológica en terreno y estadística multivariada de clasificación y ordenación con una tabla inicial de 30 censos levantados, 10 en cada uno de los tres predios prospectados. La mayoría de las especies vegetales son nativas presentándose sólo una introducida, Festuca rubra L. de origen europeo y el espectro biológico de ellas es típico de condiciones extremas. Por metodología fitosociológica tradicional se encontraron diferencias florísticas entre los censos de los tres predios que se mantienen en los arreglos estadísticos con escasas variaciones. Con la ordenación de las especies se lograron identificar como muy importante las variaciones de humedad y con menor importancia, la acumulación de materia orgánica en el sustrato. Carex kingii R. Br. y Tetroncium magellanicum Willd. aparecen como indicadores de humedad mientras que Nanodea muscosa Banks, Juncus scheuchzerioides Gaudich. y Festuca rubra, de sequía. Marssippospermum grandiflorum L.f. y Nothofagus antarctica G. Forst. se presentan ocupando posiciones intermedias. Se concluye que los predios presentan diferentes condiciones de humedad probablemente por drenaje de origen antrópico, que regula la degradación de la materia orgánica. El predio con mayor alteración se presentó como más favorable para la explotación de Sphagnum magellanicum Brid.

Palabras clave: Patagonia chilena, vegetación turbosa, fitosociología, ordenación, clasificación. 


\section{INTRODUCCIÓN}

Las turberas esfagnosas secundarias antropogénicas del Sur de Chile, dominadas ampliamente por el musgo Sphagnum magellanicum Brid., comúnmente pon-pon, son abundantes en las Regiones de Los Lagos, de Aysén y de Magallanes (Pisano, 1983; San Martín et al., 2004; Luebert y Pliscoff, 2006). Se trata de turberas topogénicas que reciben agua del suelo por encontrarse normalmente, en valles junto a cursos de agua. En años secos son colonizados por hierbas, arbustos y árboles, pero en años húmedos vuelven a recuperar su territorio. Al parecer esta dinámica motivada por variaciones climáticas o por alteración antrópica, son las que originan estos turbales esfagnosos, que invaden bosques muertos por fuego, anegamiento, explotación maderera o por acidificación del sustrato. Aunque existen turberas esfagnosas primarias, la mayoría de ellas son secundarias, ocupando el territorio de bosques húmedos chilotes de Nothofagus nitida Phil. (coihue de Chiloé) y, principalmente de Pilgerodendron uviferum D. Don (ciprés de las Guaitecas) que han sido alterados o destruidos por la acción humana (Díaz et al., 2008). Como comunidades primarias los turbales de pon-pon se forman en suelos anegados con características de ñadi o turbosos en torno a lagunas o lagos, colonizados en primera instancia por Nothofagus antarctica G. Forst (ñirre o ñire) en su forma arborescente o por Tepualia stipularis Hook. \& Arn. (tepu) en su forma arbustiva (Ramírez et al., 1993). Estas turberas primarias son frecuentes en las islas de Aisén (Álvarez et al., 2010). Las turberas ombrogénicas de Sphagnum magellanicum que pierden contacto con el sustrato mineral, son escasas en Chile, excepto en Tierra del Fuego, donde la turba presentan mayor profundidad. Una detallada revisión sobre los humedales magallánicos, incluida las turberas, entrega Promis (2010).

El musgo pon-pon y más escasamente la turba bajo él, son explotados a veces de manera poco racional como retenedores de humedad el primero y, como combustible la segunda. En Chile el musgo pon-pon es explotado en la región de Los Lagos y la turba, en la región de Magallanes, pero en la región de Aysén las turberas esfagnosas permanecen aún (año 2012) sin explotación, por lo que están muy bien conservadas (Díaz et al., 2008; Zegers et al., 2006). Lo que se ha explotado allí son los bosques primitivos, permitiendo la colonización del musgo. Pero esta explotación de madera se ha hecho tanto por tala, como por fuego y en diferentes épocas, lo que hace que las turberas resultantes sean diferentes en su composición florística, aún cuando pertenezcan a una sola comunidad (asociación vegetal) el Gaultherio-Sphagnetum magellanicii descrita por Oberdorfer (1970) (Amigo et al., 2017).

El presente estudio compara la composición florística de tres turberas esfagnosas sometidas a diversos usos antrópicos, bajo el supuesto de que especies in- dicadoras de dichos sitios (sensu Dufrene y Legendre, 1997) podrían ayudar a inferir características ecológicas derivadas de estos usos. Esta aproximación ecológica con especies indicadoras (Diekmann, 2003) ha sido utilizada recurrentemente en comunidades secundarias antropogénicas (San Martín et al., 2004) como las praderas, en donde la flora alóctona presente en ellas ha probado tener un carácter indicador de condiciones ambientales antrópicas (por ejemplo, requerimientos de sitio, compactación del suelo, sequía edáfica (Álvarez et al., 2013). Las turberas esfagnosas, sin embargo, carecen usualmente de flora exótica debido a sus condiciones extremas de anegamiento, acidez y anoxia (Teneb et al., 2008). Así, las turberas esfagnosas, representan un interesante modelo para evaluar especies nativas como indicadoras de perturbación humana. En este estudio nos planteamos las siguientes preguntas: ¿pueden las especies de plantas nativas ser indicadores ecológicos de perturbación antrópica en turberas esfagnosas? Si es así ¿qué especies nativas son indicadoras de diferentes manejos en turberas? Planteamos que estas preguntas podrían ser de interés en conservación y manejo de turberas esfagnosas, en áreas en las que estos ecosistemas son de importancia ecológica y económica, como la Región de Aisén.

\section{MATERIAL Y MÉTODOS}

\section{Lugar de trabajo}

Se trabajó en tres turberas esfagnosas ubicadas dos en la comuna de Tortel y una, en la parte sur de la comuna de Cochrane, en los predios de Don José Iñiguez y Don Hernán Guelet los primeros y de Doña Carmen Guzmán, el último. El primer predio se encuentra en el valle del río Baker cerca de Caleta Tortel junto al camino de dicha caleta a río Vagabundo. El segundo en el valle del desagüe del Lago Quetru, en la ruta en construcción al Ventisquero Montt y el tercero, en el valle del río Baker, pero más al Norte, en la comuna de Cochrane entre los ríos Vagabundo y Correntoso (Figura 1). La primera turbera, originada sobre un cipresal muerto se encuentra actualmente en explotación intensiva lo que ha favorecido la colonización de Marsippospermum grandiflorum, que se ha hecho muy abundante. La segunda turbera, con una inundación más prolongada, también se formó sobre un cipresal muerto, el cual ha sido explotado en forma moderada. La tercera turbera, muy accesible, grande y al parecer muy antigua, se desarrolló sobre un cipresal muerto, cuya madera en pie no ha sido explotada. Lo anterior le otorga características distintas a los otros turbales, ya que en ellos domina ampliamente Sphagnum magellanicum, siendo las especies acompañantes de menor importancia.

La estación meteorológica más cercana a Caleta Tortel es la Isla San Pedro ubicada a $47^{\circ} 43^{\prime}$ latitud Sur y 
$74^{\circ} 55^{\prime}$ longitud Oeste, en la bahía Tarn al Noreste de la Isla Wager en el archipiélago de Guayaneco, encontrándose casi frente a Tortel. Allí la temperatura promedio anual alcanza a $8,8^{\circ} \mathrm{C}$, la precipitación llega a $4.294 \mathrm{~mm}$ al año, correspondiendo a un bioclima supratemperado y a un ombrotipo ultraperhúmedo según Amigo y Ramírez (1998). El diagrama climático ombrotérmico de Hajek y Di Castri (1975) muestra que en todo el año la precipitación es mayor que la evaporación, y por lo tanto, no existe estación seca (Figura 1).

\section{Toma de datos en terreno}

La toma de datos en terreno se realizó entre los días 15 y 20 de octubre de 2007, levantándose 30 censos de vegetación con la metodología fitosociológica de Braun-Blanquet (1979) desarrollada en el Sur de Europa, en tres turbales esfagnosos de color rojizo, dominados por musgo pon-pon (Sphagnum magellanicum) (Dengler et al., 2008). Estos censos se hicieron en parcelas florística, fisonómica y ecológicamente homogéneas. Se levantaron 10 censos en cada uno de los sitios de estudio. Los censos se tomaron en parcelas de $100 \mathrm{~m}^{2}(10 \mathrm{~m} \times 10 \mathrm{~m})$ superiores al área mínima establecidas para asociaciones vegetales de turberas (Dierschke, 1994). En cada censo se confeccionó primero una lista de las especies vegetales presentes y luego, se determinó la abundancia de los individuos de cada una, expresándola directamente en porcentaje de cobertura de la parcela (Knapp, 1984). Además, se usaron los signos "+" y "r" (cruz y erre), para indicar coberturas bajo $1 \%$, la cruz cuando habían varios individuos y la erre, cuando sólo apareció un individuo de la especie en cuestión. Estos últimos se subieron a la unidad para cálculos posteriores. Los censos se distribuyeron por la superficie de las turberas tratando de abarcar toda la variación existente en la vegetación. En la mayoría de las parcelas la suma de los porcentajes de cobertura supera el $100 \%$, indicando una cubierta vegetal continua y a veces estratificada.

Los 30 censos de vegetación se reunieron en una sola tabla fitosociológica inicial, en la cual se determinó primeramente la flora de las turberas esfagnosas, representada en la primera columna. Se determinaron las especies desconocidas, se revisó la nomenclatura usada en terreno, se estableció la sinonimia y se clasificaron las especies utilizando la literatura taxonómica pertinente (San Martín et al., 2004; Rodríguez et

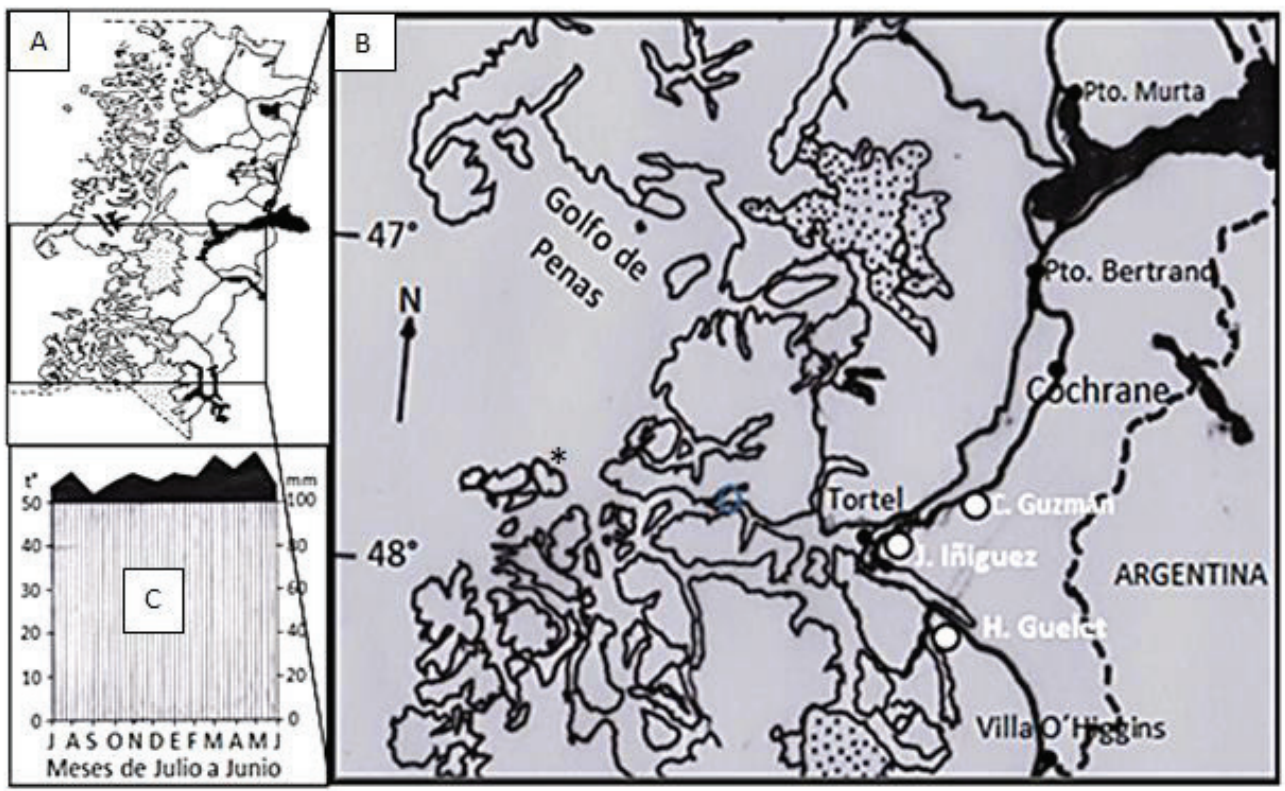

Figura 1. A = Región de Aisén del General Carlos Ibañez del Campo, Chile. $B$ = Ubicación de los lugares de muestreo (círculos blancos) en las cercanías de Caleta Tortel. C = Diagrama climático de Isla San Pedro junto a la Isla Wager que se señala con un asterisco, según Hajek y Di Castri (1975). El espacio negro del diagrama climático señala el exceso de precipitación en el lugar de trabajo, donde no hay meses secos durante todo el año.

Figure 1. $A=$ Aisén Region of General Carlos Ibañez del Campo, Chile. $B=$ Location of the sampling sites (white circles) in the vicinity of Caleta Tortel. C = Climate diagram of San Pedro Island next to Wager Island that is marked with an asterisk, according to Hajek and Di Castri (1975). The black space of the climate diagram indicates that in the workplace there are no dry months during the year. 
al., 2008) y el sitio "The plant list" (www.theplantlist. org) del Jardín botánico de Missouri. Para cada especie se determinó la forma de vida de acuerdo a MuellerDombois y Ellenberg (1974) y el origen fitogeográfico (nativa o introducida) según Zuloaga et al. (2008). Se establecieron las siguientes formas de vida según Frey y Lösch (2010): Fanerófitos (árboles, arbustos y trepadoras leñosas), caméfitos (subarbustos y hierbas altas), hemicriptófitos (hierbas perennes), criptófitos (plantas acuáticas, palustres y geófitos) y terófitos (plantas anuales). Musgos y líquenes, que tienen sus propias formas de vida, se agregaron en conjunto como formas de vida adicionales.

\section{Proceso de tabulación}

La tabla inicial se analizó en modo vertical y horizontal, en primer caso se obtuvo el número de especies por censo y la cobertura total de cada uno de ellos, calculando después el promedio general de especies, estos últimos cálculos se repitieron para las tres tablas parciales representantes de cada predio. En el análisis transversal se calcularon primero la frecuencia o número de censos en que la especie está presente y la cobertura total sumando las coberturas de cada especie en la tabla. La frecuencia fue expresada en porcentaje y estos se ordenaron en 5 clases de frecuencia con intervalos de $20 \%$, tal que el histograma resultante debe estar en concordancia con la homogeneidad de la tabla inicial (Knapp, 1984) que pertenece a una sola asociación o comunidad vegetal. Se calcularon la frecuencia y la cobertura relativa por especie, para luego sumarlas y establecer así el valor de importancia de cada una según Wikum y Shanholtzer (1978). Este valor cuya suma es 200 es adimensional y sirve para jerarquizar las especies de la tabla y sólo tiene validez para ella no siendo comparable con otras.

Posteriormente, se prepararon tablas parciales por cada predio para obtener un censo promedio y representativo de cada una para proceder a su comparación y establecer la similitud florística entre los predios, utilizando los índices de similitud de Jaccard (Badii et al., 2008) y de Ellenberg (Mueller-Dombois y Ellenberg, 1974), el primero sólo considera la presencia y el segundo, agrega la cobertura, denominándose índice de comunidad.

La tabla inicial fue ordenada primero en forma tradicional mediante la búsqueda de especies diferenciales que se excluyen mutuamente (Dierschke, 1994), logrando encontrar 9 de estas especies y separar con ellas 3 grupos de censos. Como se trabajaba con una sola asociación vegetal claramente delimitada esperábamos poder utilizar los grupos formados para diferenciar el manejo recibido por la turbera en cada predio. Para comprobar la validez de esta clasificación tradicional de la tabla inicial se analizó posteriormente con métodos estadísticos multivariados de clasificación (conglomerados) y de ordenación, para visualizar la similitud florística entre los censos, los sitios y las especies. Estas clasificaciones y ordenaciones permiten inferir que condiciones de sitio determinan las diferencias florísticas entre los censos y los requerimientos de sitio de cada especie, lo que permite establecer el carácter indicador de cada una, lo que puede servir a futuro para evaluar cambios en las turberas por intervención antrópica (Ramírez et al., 2012).

\section{Análisis estadísticos multivariados}

El análisis de clasificación (o conglomerado) se llevó a cabo mediante el uso del método jerárquico flexible beta con la distancia Bray-Curtis y un parámetro $\beta=-0,25$ (McCune y Grace, 2002) previa transformación de los valores de abundancia a logaritmo log (abundancia + 1). Este procedimiento ha sido recomendado para análisis de vegetación pues entrega resultados semejantes al método de la varianza mínima pero no ofende el principio de minimización de error de la suma de cuadrados dentro de los grupos y además, conserva las propiedades del espacio multidimensional en la distribución de las unidades de muestreo (Legendre y Legendre, 1998). La línea de corte para el conglomerado resultante se estableció de manera arbitraria, teniendo en cuenta los criterios de largo de las distancias entre grupos y cantidad de información remanente (McCune y Grace, 2002).

La ordenación de censos y especies se realizó con un Análisis Multidimensional No Métrico (Non Metric Multimensional Scaling) (Clarke y Ainsworth, 1993) para obtener una configuración gráfica de las especies de plantas y las unidades de muestreo, visualizando la relación entre los sitios en función de la composición florística. Este método es ventajoso porque examina la estructura de las matrices sin asumir relaciones lineales entre las variables de estudio (es decir especies) y porque a través de una iteración, muestra las distancias ordenadas sobre cualquier tipo de transformación o relativización de los datos (McCune y Grace, 2002). Una vez definidos los grupos mediante el análisis de conglomerados se procedió a determinar las especies indicadoras de los mismos mediante el análisis de especies indicadoras (en inglés: Indicator Species Analysis) (Dufrene y Legendre, 1997). Este análisis selecciona especies indicadoras de los grupos determinados "a priori" en base a las abundancias y frecuencias de las mismas, registradas en las unidades de muestreo de los grupos, recalculadas mediante permutaciones definidas por el usuario. El análisis entrega un valor indicador ISA asociado a un grupo y el valor de probabilidad correspondiente a la asociación. Con especies indicadoras se seleccionaron solo aquellas especies cuyos valores de probabilidad fueron estadísticamente significativos 
$(\mathrm{p}<0,05)$. ISA se calculó utilizando el paquete labdsv indval Package for R statistics software.

\section{RESULTADOS}

\section{Flora}

En los 30 censos levantados en las turberas de $S$. magellanicum se encontraron 26 especies de plantas vasculares, el líquen Cladonia pycnoclada Nyl. y el musgo dominante en turberas Sphagnum magellanicum y varios musgos no conocidos los que fueron tratados como una sola especie en las tablas fitosociológicas. Los últimos se denominaron Musgos. Mayores detalles botánicos sobre las especies se encuentran en el Cuadro 1.

De las 28 especies presentes en los censos sólo una es introducida en Chile, Festuca rubra de origen europeo. En plantas vasculares aparecen 2 helechos, Blechnum magellanicum Mett. y Blechnum penna-marina Poir. y una Conífera, Pilgerodendron uviferum. En las 23

Cuadro 1. Caracterización de las especies vegetales de las tres turberas esfagnosas estudiadas.

Table 1. Characterization of the plant species of the three peat bogs studied.

\begin{tabular}{|c|c|c|c|c|c|}
\hline Especie / Autor & Gr. & Familia & Nombre común & Or. & F.V. \\
\hline Baccharis patagonica Hook. \& Arn. & $\mathrm{D}$ & Asteraceae & Romerillo, Chilca & $\mathrm{N}$ & $\mathrm{F}$ \\
\hline Berberis ilicifolia $\mathrm{L} . \mathrm{f}$. & $\mathrm{D}$ & Berberidaceae & Chelia, Michay & $\mathrm{N}$ & $\mathrm{F}$ \\
\hline Blechnum magellanicum (Desv.) Mett. & $\mathrm{H}$ & Blechnaceae & Catalapi & $\mathrm{N}$ & $\mathrm{F}$ \\
\hline Blechnum penna-marina (Poir.) Kuhn & $\mathrm{H}$ & Blechnaceae & Punke & $\mathrm{N}$ & $\mathrm{H}$ \\
\hline Carex kingii (Boott) Reznicek & M & Cyperaceae & No conocido & $\mathrm{N}$ & $\mathrm{H}$ \\
\hline Carex magellanica Lam. & M & Cyperaceae & Cortadera negra & $\mathrm{N}$ & $\mathrm{H}$ \\
\hline Chilliotrichum diffusum (G. Forst.) Kuntze & $\mathrm{D}$ & Asteraceae & Fascine & $\mathrm{N}$ & $\mathrm{F}$ \\
\hline Cladonia pycnoclada (Pers.) Leight & $\mathrm{L}$ & Cladoniaceae & Líquen & $\mathrm{N}$ & $\mathrm{L}$ \\
\hline Drimys winteri J. R. Forst. \& G. Forst. & $\mathrm{D}$ & Winteraceae & Canelo, Voige & $\mathrm{N}$ & $\mathrm{F}$ \\
\hline Empetrum rubrum Vahl ex Willd. & $\mathrm{D}$ & Empetraceae & Brecillo & $\mathrm{N}$ & $\mathrm{C}$ \\
\hline Escallonia virgata (Ruiz \& Pav.) Pers. & $\mathrm{D}$ & Escalloniaceae & Chapel, Meki & $\mathrm{N}$ & $\mathrm{F}$ \\
\hline Festuca rubra L. & M & Poaceae & Coirón & I & $\mathrm{H}$ \\
\hline Festuca pyrogea Speg. & $\mathrm{M}$ & Poaceae & Coirón & $\mathrm{N}$ & $\mathrm{H}$ \\
\hline Gaultheria antarctica Hook. f. & $\mathrm{D}$ & Ericaceae & Chaurilla & $\mathrm{N}$ & $\mathrm{C}$ \\
\hline Gaultheria mucronata (L. f.) Hook. \& Arn. & $\mathrm{D}$ & Ericaceae & Chaura & $\mathrm{N}$ & $\mathrm{C}$ \\
\hline Gaultheria pumilla (L. f.) B. J. Middleton & $\mathrm{D}$ & Ericaceae & No conocido & $\mathrm{N}$ & $\mathrm{C}$ \\
\hline Juncus Scheuchzerioides Gaudich, & M & Juncaceae & No conocido & $\mathrm{N}$ & $\mathrm{H}$ \\
\hline Marsippospermum grandiflorum (L. f.) Hook. & M & Juncaceae & Junco canasto & $\mathrm{N}$ & $\mathrm{H}$ \\
\hline Myrteola nummularia (Poir.) O. Berg. & $\mathrm{D}$ & Myrtaceae & Haurapo, daudapo & $\mathrm{N}$ & $\mathrm{C}$ \\
\hline Nanodea muscosa Banks. \& C. F. Gaertn. & $\mathrm{D}$ & Santalaceae & No conocida & $\mathrm{N}$ & $\mathrm{H}$ \\
\hline Nothofagus antarctica (G. Forst.) Oerst. & $\mathrm{D}$ & Nothofagaceae & Ñire, Ñirre & $\mathrm{N}$ & $\mathrm{F}$ \\
\hline Nothofagus betuloides (Mirb.) Oerst. & $\mathrm{D}$ & Nothofagaceae & Coihue de Magallanes & $\mathrm{N}$ & $\mathrm{F}$ \\
\hline Philesia magellanica J. F. Gmel. & $\mathrm{M}$ & Philesiaceae & Coicopihue & $\mathrm{N}$ & $\mathrm{F}$ \\
\hline Pilgerodendron uviferum (D. Don) Florin & $\mathrm{C}$ & Cupressaceae & Ciprés de las Guaitecas & $\mathrm{N}$ & $\mathrm{F}$ \\
\hline Raukaua laetevirens (Gay) Frodin & $\mathrm{D}$ & Araliaceae & Sauquillo & $\mathrm{N}$ & $\mathrm{F}$ \\
\hline Rubus geoides $\mathrm{Sm}$. & $\mathrm{D}$ & Rosaceae & Miñe-Miñe & $\mathrm{N}$ & $\mathrm{C}$ \\
\hline Sphagnum magellanicum Brid. & Ms & Sphagnaceae & Pon-Pon & $\mathrm{N}$ & Ms \\
\hline Tetroncium magellanicum Willd. & M & Juncaginaceae & No conocido & $\mathrm{N}$ & $\mathrm{H}$ \\
\hline
\end{tabular}

Abreviaturas: $\mathrm{Gr}$ = grupo sistemático ( $\mathrm{L}=$ Líquenes, $\mathrm{Ms}=$ Musgos, $\mathrm{H}=$ Helechos, $\mathrm{G}=$ Gimnospermas, $\mathrm{D}=$ Dicotiledóneas (en sentido amplio), $\mathrm{M}=$ Monocotiledóneas), Or. = Origen fitogeográfico ( $\mathrm{N}=$ nativo, $\mathrm{I}=$ introducido), $\mathrm{FV} . \mathrm{l}=$ forma de vida $(\mathrm{F}=$ fanerófitos, $\mathrm{C}=$ caméfitos, $\mathrm{H}=$ hemicriptófitos $)$. 
Angiospermas restantes figuran $8(28,6 \%)$ Monocotiledóneas, siendo el grupo dominante, y 15 (53,6\%) especies de Dicotiledóneas (Cuadro 2).

El espectro biológico de esta flora turbosa es muy reducido ya que sólo presenta 3 formas de vida, fanerófitos (plantas leñosas), caméfitos (subarbustos) y hemicriptófitos (hierbas perennes), faltando las formas de vida criptofítica (geófitos) y terofítica, que son propias de lugares secos. Los fanerófitos presentaron $11(39,3 \%)$ especies, los caméfitos $6(21,4 \%)$ y los hemicriptófitos $9(32,1 \%)$ especies (Cuadro 2$)$.

\section{Vegetación}

Del análisis de la tabla inicial se desprende que el promedio de especies por censo alcanzó a 9,3 con un mínimo de 5 y un máximo de 16 especies por censo. En cada predio este promedio varió como se señala en el Cuadro 3, presentando los predios Iñiguez y Guzmán el mismo promedio de 9,5 especies por censos, pero con una diferencia entre máxima y mínima de 8 el primero y 4 el segundo. El promedio más bajo lo presentó el predio Guelet con 8,9 especies y una diferencia de 4 . Esto señala al Predio Guzmán como más estable y florísticamente más homogéneo.

En un análisis transversal de la tabla, la única especie presente en todos los censos (30) es Sphagnum magellanicum, seguido por Gaultheria mucronata L.f.

Cuadro 2. Distribución de la flora en grandes grupos sistemáticos y por formas de vida.

Table 2. Distribution of the flora in large systematic groups and by life forms.

\begin{tabular}{lcc}
\hline Grupo & Especies & Porcentaje \\
\hline Líquenes & 1 & 3,6 \\
Musgos & 1 & 3,6 \\
Helechos & 2 & 7,1 \\
Coníferas & 1 & 3,6 \\
Monocotiledóneas & 8 & 28,6 \\
Dicotiledóneas & 15 & 53,6 \\
\hline Total & $\mathbf{2 8}$ & $\mathbf{1 0 0}$ \\
\hline Formas de Vida & & \\
Fanerófitos & 11 & 39,3 \\
Caméfitos & 6 & 21,4 \\
Hemicriptófitos & 9 & 32,1 \\
Líquen & 1 & 3,6 \\
Musgo & 1 & 3,6 \\
\hline Total & $\mathbf{2 8}$ & $\mathbf{1 0 0}$ \\
\hline
\end{tabular}

presente en 26, Nothofagus betuloides Mirb. en 25, Marsippospermum grandiflorum en 23 y Empetrum rubrum Vahl. en 20 censos (Cuadro 4). La mayor cobertura al sumar todos los censos donde la especie está presente, correspondió a Sphagnum magellanicum con $2.438 \%$, dominando absolutamente en la vegetación estudiada. Le siguen Marsippospermum grandiflorum con $584 \%$ y Empetrum rubrum con $220 \%$. Al ordenar las especies por su importancia en la tabla inicial, la más importante resultó también Sphagnum magellanicum $(71,8)$, seguida de Marsippospermum grandiflorum $(22,9)$, Gaultheria mucronata $(14,3)$, Empetrum rubrum $(12,7)$ y Blechnum penna-marina y Nothofagus betuloides con 10,9 y 10,7 , respectivamente.

Los datos anteriores confirman el hecho de que todos los censos pertenecen a la misma comunidad vegetal, la cual resulta muy homogénea al ordenar las frecuencias (presencia) por clases (Figura 2), donde se observan valores superiores a la clase siguiente y anterior, en la primera y última clase, proporción que corresponde a una asociación vegetal (comunidad vegetal) bien definida y estructurada.

No obstante lo anterior, al ordenar en forma tradicional la tabla fitosociológica inicial, entre los tres

Cuadro 3. Riqueza florística de la turbera de cada predio.

Table 3. Floristic richness of the peat bog of each property.

Predio /Número Promedio Máximo Mínimo Diferencia de especies:

\begin{tabular}{lllll}
\hline José Iñiguez & 9,5 & 16 & 8 & 8 \\
Hernán Guelet & 8,9 & 13 & 5 & 8 \\
Carmen Guzmán & 9,5 & 10 & 6 & 4 \\
\hline
\end{tabular}

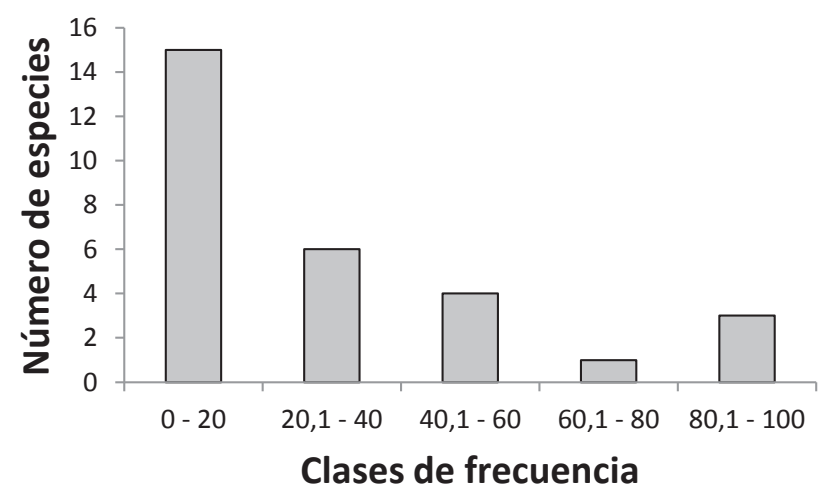

Figura 2. Diagrama de Frecuencia de las especies de la Tabla inicial.

Figure 2. Frequency diagram of the species in the initial Table. 
predios se ven diferencias, que están reflejadas en la presencia o ausencia de determinadas especies en cada predio. El Cuadro 5 indica en la parte superior estas diferencias, en él se aprecia que el predio Iñiguez presenta una dominancia de Marsippospermum grandiflorum y de Blechnum penna-marina y ausencia de 5 de las 6 especies que diferencian a los otros dos predios, Carex kingii, Tetroncium magellanicum, Juncus scheuchzerioides, Nanodea muscosa y Festuca rubra. Lo anterior constituye una gran diferencia en la composición florística del primer predio. En el predio Guelet, están presentes en forma exclusiva Carex kingii y Tetroncium magellanicum aunque faltan 6 especies diferenciales de los otros predios y también Nothofagus antarctica y Gaultheria

Cuadro 4. Análisis estadístico transversal de la tabla inicial ordenada por valor de importancia.

Table 4. Cross-sectional statistical analysis of the initial table ordered by importance value.

\begin{tabular}{|c|c|c|c|c|c|c|}
\hline Especies / Censos: & Fr. & $\% \mathrm{Fr}$. & Cob. & Fr.rel & Cob.rel & V.I. \\
\hline Sphagnum magellanicum & 30 & 100 & 2438 & 10,6 & 61,1 & 71,8 \\
\hline Marsippospermum grandiflorum & 23 & 76,7 & 584 & 8,2 & 14,6 & 22,9 \\
\hline Gaultheria mucronata & 26 & 86,7 & 198 & 9,3 & 5,0 & 14,3 \\
\hline Empetrum rubrum & 20 & 66,7 & 220 & 7,2 & 5,5 & 12,7 \\
\hline Blechnum penna-marina & 18 & 60,0 & 177 & 6,5 & 4,4 & 10,9 \\
\hline Nothofagus betuloides & 25 & 83,3 & 70 & 8,0 & 1,8 & 10,7 \\
\hline Pilgerodendron uviferum & 14 & 46,7 & 91 & 5,0 & 2,3 & 7,3 \\
\hline Gaultheria pumila & 13 & 43,3 & 13 & 4,7 & 0,3 & 5,0 \\
\hline Festuca pyrogea & 12 & 40,0 & 14 & 4,3 & 0,4 & 4,7 \\
\hline Nothofagus antarctica & 12 & 40,0 & 12 & 4,3 & 0,3 & 4,6 \\
\hline Berberis ilicifolia & 9 & 30,0 & 22 & 3,2 & 0,6 & 3,8 \\
\hline Juncos scheuchzerioides & 8 & 26,7 & 25 & 2,9 & 0,6 & 3,5 \\
\hline Carex kingii & 6 & 20,0 & 43 & 2,2 & 1,1 & 3,2 \\
\hline Festuca rubra & 6 & 20,0 & 25 & 2,2 & 0,6 & 2,8 \\
\hline Chiliotrichum diffusum & 7 & 23,3 & 7 & 2,5 & 0,2 & 2,7 \\
\hline Blechnum magellanicum & 7 & 23,3 & 7 & 2,5 & 0,2 & 2,9 \\
\hline Rubus geoides & 6 & 20,0 & 6 & 2,2 & 0,2 & 2,3 \\
\hline Tetroncium magellanicum & 6 & 20,0 & 6 & 2,2 & 0,2 & 2,3 \\
\hline Cladonia pycnoclada & 5 & 16,7 & 5 & 1,8 & 0,1 & 1,9 \\
\hline Raukaua laetevirens & 5 & 16,7 & 5 & 1,8 & 0,1 & 1,9 \\
\hline Philesia magellanica & 4 & 13,3 & 4 & 1,4 & 0,1 & 1,5 \\
\hline Myrteola nummularia & 4 & 13,3 & 4 & 1,4 & 0,1 & 1,5 \\
\hline Escallonia virgata & 3 & 10,0 & 3 & 1,1 & 0,1 & 1,2 \\
\hline Nanodea muscosa & 3 & 10,0 & 3 & 1,1 & 0,1 & 1,2 \\
\hline Carex magellanica & 2 & 6,7 & 6 & 0,7 & 0,2 & 0,9 \\
\hline Musgos & 2 & 6,7 & 2 & 0,7 & 0,1 & 0,8 \\
\hline Gaultheria antarctica & 1 & 3,3 & 1 & 0,4 & 0,0 & 0,4 \\
\hline Baccharis patagonica & 1 & 3,3 & 1 & 0,4 & 0,0 & 0,4 \\
\hline Drimys winteri & 1 & 3,3 & 1 & 0,4 & 0,0 & 0,4 \\
\hline Totales: & 279 & & 3993 & 100 & 100 & 200 \\
\hline
\end{tabular}

Abreviaciones: Fr. = frecuencia, \% Fr. = Porcentaje de frecuencia, Cob. Cobertura total, Fr.re = Frecuencia relativa, Cob.rel = cobertura relativa V.I. = Valor de importancia según Wikum y Shanholtzer (1978). 
pumila L.f. (en Cuadro 5 espacio sombreado). Por último, el predio Guzmán presenta tres especies exclusivas Juncus scheuchzerioides, Nanodea muscosa y Festuca rubra, esta última, la única especie alóctona de la tabla fitosociológica inicial.

Comparando ahora los 3 censos promedio que resultan de cada predio, formando una tabla resumida (Cuadro 6) se aprecia claramente que el predio Guelet se diferencia de los otros 2 al presentar el mayor número de especies exclusivas (4) y también la mayor riqueza de especies (23). En la misma tabla se observa que los tres predios comparten 12 especies, la mayoría de ellas con alta cobertura y que son las que caracterizan y diferencian la asociación vegetal, además hay 9 especies compartidas por dos predios y 8 que sólo están presentes o son exclusivas de uno. Los predios fueron comparados florísticamente en esta misma tabla, observándose una similitud florística de los tres predios entre 50 y $60 \%$ al utilizar sólo la presencia de especies en cada uno. Pero al considerar la cobertura promedio de las especies estos valores suben en torno a un $90 \%$. Lo anterior demuestra que las diferencias florísticas entre los predios son apreciables, pero las especies do- minantes compartidas determinan una similitud muy alta, que reafirma la presencia de una sola comunidad en la tabla ordenada.

\section{Ordenación y clasificación multivariable}

El análisis de clasificación (conglomerados) muestra que pueden considerarse 3 grupos, pero el único que no sufre cambios es el predio Guelet que mantiene sus 10 censos, mientras que el predio Iñiguez aparece con 12 censos, de los cuales 2 son sacados del predio Guzmán, que sin embargo, resulta ser el más aislado de los tres, ya que los predios Iñiguez y Guelet están unidos entre ellos con mayor similitud (Figura 3). Los censos retirados del predio Guzmán corresponden al 21 y 22 que presentan Marsippospermum grandiflorum y carecen de las otras especies diferenciales del predio como Juncus scheuchzerioides, Nanodea muscosa y Festuca rubra.

El análisis de ordenación, distribuyendo las especies y los censos en el plano formado por los dos primeros ejes del análisis que son los más importantes, muestra que los 8 censos del predio Guzmán se presentaron muy juntos formando una unidad coheren-

Cuadro 5. Tabla ordenada mostrando las especies diferenciales de cada predio.

Table 5. Ordered table showing the differential species of each property.

\begin{tabular}{|c|c|c|c|c|c|c|c|c|c|c|c|c|c|c|c|}
\hline \multirow{2}{*}{$\begin{array}{l}\text { Predios } \\
\text { Especies / Censos: }\end{array}$} & \multicolumn{10}{|c|}{ Predio1 José Iñiguez } & \multicolumn{5}{|c|}{ Predio 2 Hernán } \\
\hline & 1 & 2 & 3 & 4 & 5 & 6 & 7 & 8 & 9 & 10 & 11 & 12 & 13 & 14 & 15 \\
\hline \multicolumn{16}{|l|}{ Especies diferenciales } \\
\hline Berberis iliifolia & & 1 & 1 & 1 & & 10 & 1 & 1 & 1 & & & & & & \\
\hline Mrasippospermum grandiflorum & 40 & 50 & 40 & 40 & 50 & 40 & 60 & 40 & 40 & 40 & & 5 & 1 & 15 & 5 \\
\hline Blechnum penna-marina & 10 & 20 & 15 & 1 & 20 & 15 & 15 & 20 & 20 & 20 & & 10 & & & \\
\hline Carex kingii & & & & & & & & & & & & 20 & & 5 & 1 \\
\hline Tetroncium magellanicum & & & & & & & & & & & & & 1 & 1 & \\
\hline Empetrum rubrum & 5 & 1 & 20 & 10 & 1 & & 10 & & & 1 & 1 & & 15 & & \\
\hline \multicolumn{16}{|l|}{ Juncus scheuchzerioides } \\
\hline \multicolumn{16}{|l|}{ Nanodea muscosa } \\
\hline \multicolumn{16}{|l|}{ Festuca rubra } \\
\hline \multicolumn{16}{|l|}{ Especies características } \\
\hline Sphagnum magellanicum & 70 & 70 & 80 & 80 & 60 & 60 & 70 & 70 & 80 & 80 & 99 & 80 & 90 & 80 & 90 \\
\hline Gaultheria mucronata & 5 & 5 & 1 & 15 & 10 & 10 & 1 & 5 & 5 & 1 & 30 & 5 & 5 & 10 & 15 \\
\hline Nothofagus betuloides & 1 & 1 & 1 & 1 & 1 & 1 & 5 & 1 & 1 & 1 & 1 & & 15 & 15 & 1 \\
\hline Pilgerodendron uviferum & 1 & & & & & 5 & 10 & & & & & 5 & 1 & & \\
\hline Festuca pyrogea & & & & & & & 1 & & & 1 & 1 & 1 & & 1 & 1 \\
\hline Nothofagus antarctica & & & & 1 & 1 & 1 & 1 & & 1 & & & & & & \\
\hline Gaultheria pumila & 1 & 1 & 1 & & & & 1 & 1 & 1 & & & & & & \\
\hline Chiliotrichum diffusum & & 1 & & & & 1 & 1 & 1 & & & & 1 & & & \\
\hline
\end{tabular}




\begin{tabular}{|c|c|c|c|c|c|c|c|c|c|c|c|c|c|c|c|}
\hline \multirow{2}{*}{$\begin{array}{l}\text { Predios } \\
\text { Especies / Censos: }\end{array}$} & \multicolumn{5}{|c|}{ Guelet } & \multicolumn{10}{|c|}{ Predio 3 Carmén Guzmán } \\
\hline & 16 & 17 & 18 & 19 & 20 & 21 & 22 & 23 & 24 & 25 & 26 & 27 & 28 & 29 & 30 \\
\hline \multicolumn{16}{|l|}{ Especies diferenciales } \\
\hline Berberis iliifolia & 5 & & & & 1 & & & & & & & & & & \\
\hline Mrasippospermum grandiflorum & 15 & 1 & 20 & 10 & 10 & 40 & 20 & & & & 1 & 1 & & & \\
\hline Blechnum penna-marina & 1 & & 5 & & 1 & 1 & 1 & & & & & & & 1 & 1 \\
\hline Carex kingii & & & 1 & 1 & 15 & & & & & & & & & & \\
\hline Tetroncium magellanicum & 1 & 1 & 1 & 1 & & & & & & & & & & & \\
\hline Empetrum rubrum & & & 1 & & & 20 & 10 & 30 & 10 & 15 & 10 & 20 & 20 & 15 & 5 \\
\hline Juncus scheuchzerioides & & & & & & & & 10 & 1 & 5 & 1 & 1 & 5 & 1 & 1 \\
\hline Nanodea muscosa & & & & & & & & 1 & 1 & 1 & & & & & \\
\hline Festuca rubra & & & & & & & & & & 10 & 5 & 1 & 5 & 1 & 3 \\
\hline \multicolumn{16}{|l|}{ Especies características } \\
\hline Sphagnum magellanicum & 80 & 90 & 60 & 90 & 80 & 80 & 80 & 90 & 99 & 90 & 80 & 90 & 90 & 90 & 90 \\
\hline Gaultheria mucronata & & 20 & 20 & 10 & 5 & 10 & 1 & 1 & 5 & & 1 & 1 & 1 & & \\
\hline Nothofagus betuloides & 10 & 1 & & 5 & 1 & 1 & 1 & 1 & 1 & 1 & 1 & & & 1 & \\
\hline Pilgerodendron uviferum & 5 & & & & & 1 & 10 & 1 & & 1 & 40 & & 1 & 5 & 5 \\
\hline Festuca pyrogea & & 1 & 1 & 1 & 3 & & & 1 & & 1 & & & & & \\
\hline Nothofagus antarctica & & & & & & & 1 & 1 & 1 & 1 & 1 & & 1 & 1 & \\
\hline Gaultheria pumila & & & & 1 & & 1 & 1 & 1 & 1 & 1 & & & 1 & & \\
\hline Chiliotrichum diffusum & & & & & & & & & & & & & & 1 & 1 \\
\hline
\end{tabular}

(Faltan 12 especies con menor presencia). Los números indican porcentaje de cobertura.

Cuadro 6. Resumen de la presencia y cobertura (abundancia) promedio de las tres turberas esfagnosas estudiadas.

Table 6. Summary of the presence and coverage (abundance) average of the three peat bogs studied.

\begin{tabular}{lcccc}
\hline Especies / Predio: & Iñiguez & Guelet & Guzmán & Frecuencia \\
\hline Sphagnum magellanicum & 72 & 83,9 & 87,9 & 3 \\
Marsippospermum grandiflorum & 44 & 8,2 & 6,2 & 3 \\
Empetrum rubrum & 4,8 & 1,7 & 15,5 & 3 \\
Gaultheria mucronata & 5,8 & 12 & 2 & 3 \\
Blechnum penna-marina & 15,6 & 1,7 & 1 & 3 \\
Pilgerodendron uviferum & 1 & 1 & 6,4 & 3 \\
Nothofagus betuloides & 1 & 4,9 & 1 & 3 \\
Gaultheria pumila & 1 & 1 & 1 & 3 \\
Chiliotrichum diffusum & 1 & 1 & 1 & 3 \\
Blechnum magellanicum & 1 & 1 & 1 & 3 \\
Cladonia pycnoclada & 1 & 1 & 1 & 3 \\
Festuca pyrogea & 1 & 1 & 3 \\
Rubus geoides & 1 & 1 & 1 & 2 \\
Berberis ilicifolia & 1 & 1 & 1 & 2 \\
Philesia magellanica & & 1 & \\
\hline
\end{tabular}


Continuación Cuadro 6

\begin{tabular}{lcccc}
\hline Especies / Predio: & Iñiguez & Guelet & Guzmán & Frecuencia \\
\hline Nothofagus antarctica & 1 & & 1 & 2 \\
Escallonia virgata & 1 & & 1 & 2 \\
Carex magellanica & 1 & 1 & & 2 \\
Musgos & & 1 & 1 & 2 \\
Myrteola nummularia & & 1 & 1 & 2 \\
Raukaua laetevirens & & 4,3 & & 2 \\
Carex kingii & & & 2,5 & 1 \\
Juncos scheuchzerioides & 1 & 1 & & 1 \\
Festuca rubra & & 1 & & 1 \\
Gaultheria antarctica & & & 1 \\
Baccharis patagonica & & 23 & 1 \\
Tetroncium magellanicum & & & 1 \\
Drimys winteri & 19 & & 20 & 1 \\
Nanodea muscosa & & & 62 \\
Total & & & & 1 \\
\hline
\end{tabular}

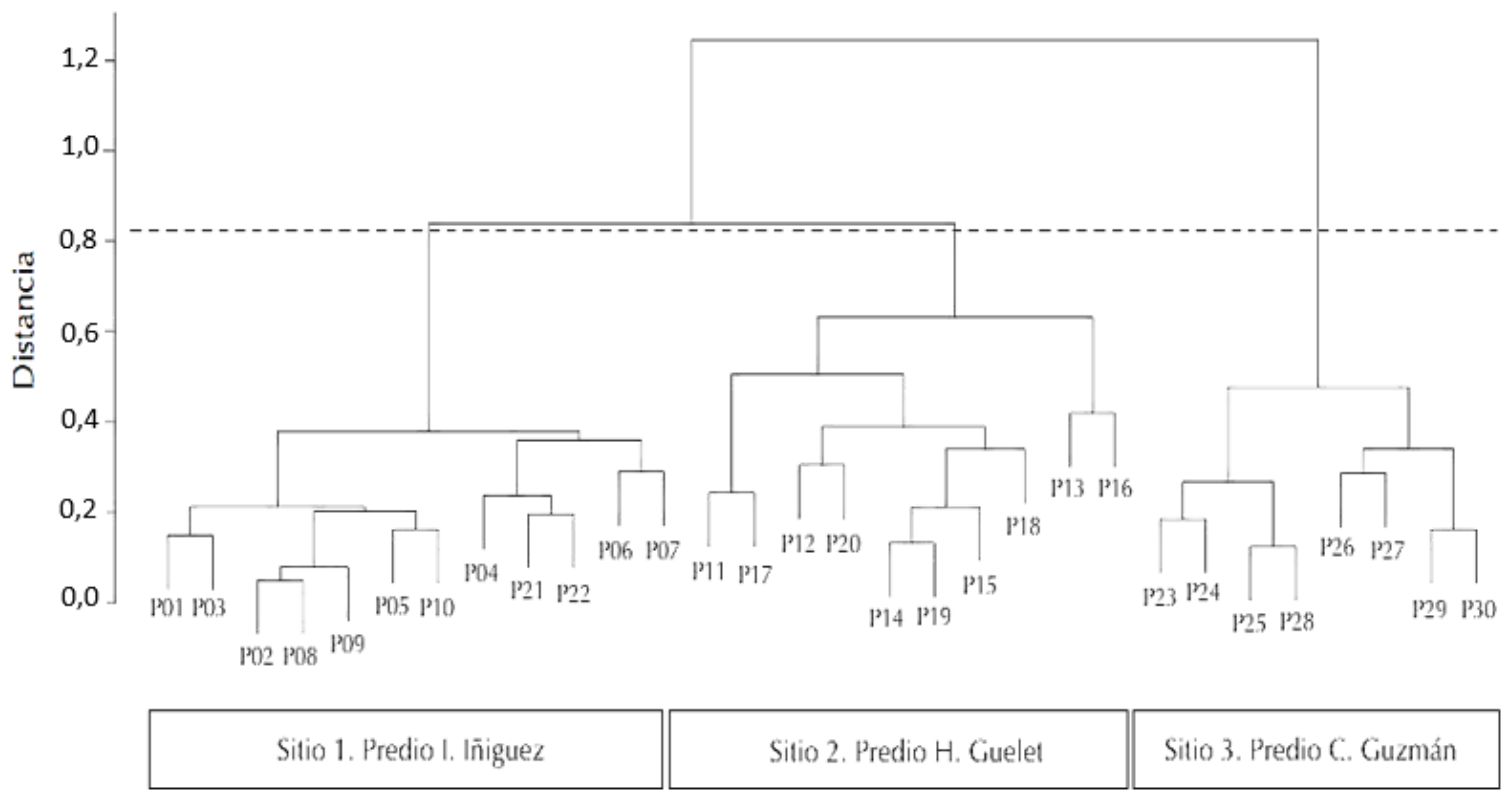

Figura 3. Dendrograma de los censos de vegetación (números) levantados en las tres turberas esfagnosas estudiadas. Se indican los predios a que corresponden los censos. Los censos P21 y P22 ubicados en el conglomerado del predio Iñiguez corresponden al predio Guzmán. La línea interrumpida indica la separación de los conglomerados.

Figure 3. Dendrogram of the vegetation samples (numbers) take in the three studied peat bogs. The properties to which the samples correspond are indicated. The samples P21 and P22 located in the conglomerate of the Iñiguez property correspond to the Guzmán site. The interrupted line indicates the separation of the conglomerates. 
te, lo mismo sucede con el predio Iñiguez, el cual sin embargo, contiene dos censos pertenecientes al predio Guzmán (Figura 4). El predio Guelet presentó una gran dispersión de sus censos ubicándose éstos mucho más cerca del predio Iñiguez, que del predio Guzmán, aún cuando hay uno de sus censos muy cerca de este último. El valor del estrés (Stress) de la ordenación fue de 13,6 indicando que los resultados en la visualización de las unidades de muestreo y las especies indicadoras, son muy estables.

Al observar la distribución de las especies indicadoras del ISA se aprecia que ellas están más segregadas por el primer eje horizontal que por el vertical. Por el primer eje se separan en el lado derecho Juncus scheuchzerioides, Nanodea muscosa y Festuca rubra, en el otro extremo (lado izquierdo) se ubica Carex kingii, Marsippospermum grandiflorum, Blechnum pennamarina y Tetroncium magellanicum, pero más cerca del centro. Una posición parecida pero en el lado derecho, ocupa Nothofagus antarctica. Por el segundo eje de ordenación, Blechnum penna-marina se ubica en la mitad superior del diagrama y Tetroncium magellani- cum y Nanodea muscosa en el lado inferior, pero todas muy cercanas al centro. La separación de Carex kingii y Tetroncium magellanicum en el lado izquierdo y de Festuca rubra, Nanodea muscosa y Juncus scheuchzerioides en el otro, permiten relacionar este eje con una gradiente de humedad (anegamiento) que aumenta de izquierda a derecha. El segundo eje es más difícil de identificar, ya que la segregación de las especies es menor, en su parte superior se ubica Blechnum pennamarina y en la inferior, Tetroncium magellanicum y $\mathrm{Na}$ nodea muscosa, sin embargo, es posible asimilarlo con un gradiente de materia orgánica o profundidad de la turba que aumentaría de abajo hacia arriba. De manera que los dos cuadrantes superiores corresponderían a un menor espesor de la turba y los dos inferiores a un mayor espesor de la misma, mientras que los dos izquierdos serían muy húmedos y los dos derechos, más secos. De acuerdo a esta interpretación en el predio Iñiguez sería seco y con menos turba, Guelet sería húmedo y turboso, mientras que Guzmán correspondería a seco con presencia de turba intermedia, entre los otros dos predios.

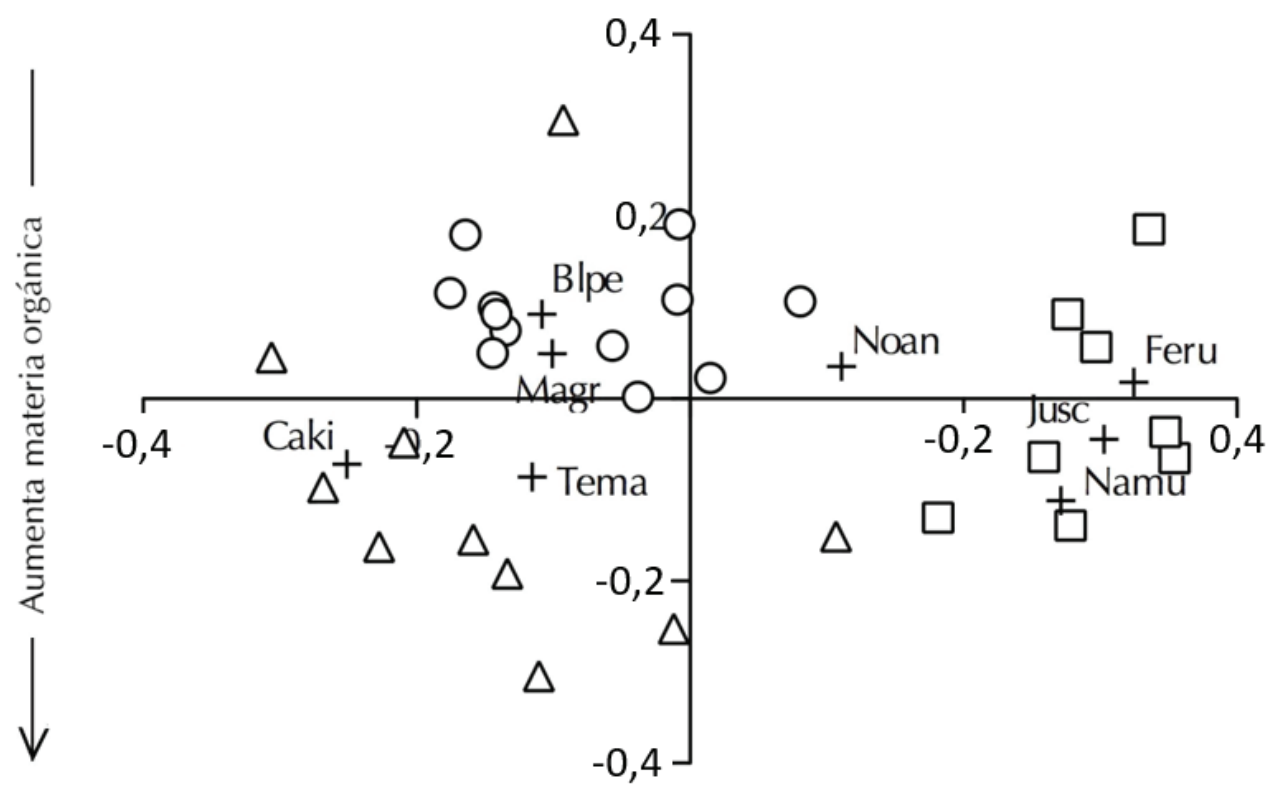

Aumenta anegamiento

Figura 4. Distribución de los censos levantados en los predios Guelet (triángulos), Iñiguez (cuadrados) y Guzmán (círculos) en los dos primeros ejes del análisis NMMS. Las cruces indican la posición de las especies más importantes cuyas abreviaturas corresponde a las dos primeras letras del sustantivo genérico y las dos primeras del adjetivo específico del nombre científico de la especie (ver Cuadro 1). Las flechas indican la dirección del aumento de los factores que representa cada uno de los dos primeros ejes del análisis de ordenación.

Figure 4. Distribution of the vegetation samples taken in the Guelet (triangles), Iñiguez (squares) and Guzmán (circles) farms in the first two axes of the NMMS analysis. The crosses indicate the position of the most important species whose abbreviations correspond to the first two letters of the generic noun and the first two of the specific adjective of the species scientific name (see Table 1). The arrows indicate the direction of the increase in the factors represented by each of the first two axes of the ordination analysis. 
Es interesante destacar que las especies situadas en los extremos de los ejes y que han servido para inferir e interpretar la variable ecológica que ellos representarían, son las mismas que fueran diferenciadas por los métodos tradicionales representados en el Cuadro 5, de estas Blechnum penna-marina (ISV $=0,853^{* *}$ ) resultó diferenciadora del predio Iñiguez, Tetroncium magellanicum (ISV $=0,755^{* * *}$ ) y Carex kingii (ISV $=0,602^{*}$ ) son diferenciales del predio Guelet y Juncus scheuchzerioides (ISV $=1,000^{* * *}$ ), Festuca rubra (ISV $=0,866^{* * *}$ ) y Nanodea muscosa (ISV $=0,612$ ) del predio Guzmán. En posición intermedia y como indicadora de los predios Iñiguez y Guelet resultó Marsippospermum grandiflorum (ISV $=0,962^{* * *}$ ). Finalmente, Nothofagus antarctica (ISV $=0,712^{*}$ ) también es indicadora para dos sitios los predios Guelet y Guzmán.

\section{DISCUSIÓN}

La flora de las turberas estudiadas pertenecientes a la asociación Gaultherio-Sphagnetum magellanicii Oberdorfer (1960) es muy pobre en especies, ya que en los 30 censos levantados en ellas sólo se encontraron 28 especies, coincidiendo con el estudio de Teneb et al. (2008), quienes reportaron 18 especies para dos turberas de Aisén. Lo anterior contrasta con la cantidad de especies que presentan por ejemplo, las turberas de la Isla de Chiloé donde con 36 muestreos Díaz et al. (2008) encontraron 74 especies de musgos, líquenes y plantas vasculares. Esta alta cantidad de especies representa seguramente una mayor intervención humana como lo plantean los autores y como lo señala la presencia de malezas alóctonas tales como Anthoxanthum odoratum L., Hypochaeris radicata L. y Lotus uliginosus Schkuhr., entre otras. Sin embargo, al muestrear un transecto es posible la inclusión de zonas ecotonales, lo que aumentaría la riqueza florística, pero con especies ajenas a la turbera. Aunque en la literatura se considera que un aumento de especies alóctonas son indicadoras de comunidades secundarias alteradas por la acción humana (San Martín et al., 1999) hay escasas excepciones como lo son las comunidades de marismas (Ramírez et al., 2018). En el caso de las turberas, esta explicación tampoco sirve porque dada las extremas condiciones del biotopo no pueden subsistir en él malezas ruderales propias de condiciones de cultivo, más favorables, lo que se demuestra con la presencia de ellas en praderas que reemplazan al bosque nativo en el mismo macroclima de las turberas, pero en suelos sin anegamiento (Walter, 1997). La forma de vida que destaca es la de caméfitos y que caracteriza a lugares extremos como son las turberas, donde faltan nutrientes minerales y también oxígeno por anegamiento (Thiers et al., 2007; Dec et al., 2017), lo que impide la oxidación de la materia orgánica del sustrato disminuyendo la actividad biológica del mismo.
En nuestro caso sólo existió una especie introducida, Festuca rubra. Tanto la pobreza en especies como la simplificación del espectro biológico de las turberas muestreadas en el presente estudio demuestran su condición de biotopos extremos. En todo caso el predio Guzmán, probablemente más seco e intervenido, presentó la menor cantidad de especies. La especie dominante Sphagnum magellanicum así como otras especies de turberas con altos valores de importancia demuestran que las tres turberas estudiadas pertenecen a la misma asociación vegetal, como también lo señala la homogeneidad de la tabla inicial plasmada en el diagrama de frecuencia (Figura 2) (Knapp, 1984). Sin embargo, con métodos tradicionales se encontraron especies que diferenciaban las distintas turberas, las que sin duda, representan distintos manejos y origen. El predio Guelet domina sobre los otros dos, tanto por el número de especies como también por el número de especies exclusivas, lo que podría corresponder a una turbera más nueva, actualmente aun en formación.

El análisis de conglomerado confirmó lo encontrado con los métodos tradicionales, pero retiró dos censos del predio Guzmán, lo que seguramente se debe a un distinto manejo local en ellos. Estos censos fueron adscritos al predio Iñiguez lo que coloca a ambos predios como más cercanos en cuanto al manejo e intervención.

$\mathrm{Al}$ analizar la ordenación multivariada de censos y especies es posible inferir una evolución del cambio florístico por el manejo que va desde el predio Guelet que sería el más joven, con la turbera aún en formación, pasando por el Predio Iñiguez para llegar al predio Guzmán que representaría el más alterado y antropizado. A juzgar por la interpretación de los ejes, es posible suponer que en esta evolución cambia preferentemente el contenido de agua del suelo seguramente por drenaje. Lo anterior se confirma con las especies Tetroncium magellanicum y Marsippospermum grandiflorum, que ocupan una posición intermedia entre los predios Guelet e Iñiguez y Nothofagus antarctica que se ubica entre el predio Iñiguez y Guzmán. Esto confirma lo planteado por Roig et al. (1983) con respecto a Marsippospermum grandiflorum. Las dos primeras especies serían indicadoras de alta humedad, mientras que el último se establecería en lugares más secos. En todo caso Nothofagus antarctica es una especie muy variable cuyos ecotipos colonizan ambientes muy diversos como lo demostraran Ramírez et al. (1985). Al observar la ordenación de los censos se ve una mayor dispersión de aquellos del predio Guelet, más cerca del original; mientras que en los otros dos predios los censos son más homogéneos al estar más próximos entre sí. Los censos retirados de Guzmán y capturados por Iñiguez, sólo indican una diferenciación local y también señalan la dirección del cambio. La dispersión de los censos del predio Guelet podría estar indicando poco manejo, pero variado. 
Estos análisis muestran que varias especies turbosas pueden ser útiles indicadoras de los cambios antropogénicos provocados en ellas. Así por ejemplo, Carex kingii y Tetroncium magellanicum serían indicadores de una mayor humedad edáfica (contenido de agua del suelo) características de los predios poco intervenidos, mientras que Nanodea muscosa, Juncus scheuchzerioides y Festuca rubra, serían indicadoras de sequía en la turbera, seguramente por un descenso del agua freática por drenaje. El segundo eje del análisis de ordenación es difícil de interpretar, pero podría tratarse de una gradiente de actividad biológica que degradaría con mayor velocidad la materia orgánica en los predios intervenidos. También el drenaje que facilita la entrada de aire al sustrato permite pasar de condiciones anaeróbicas a aeróbicas. Estas especies indicadoras son muy eficientes, ya que también diferencian los predios mediante el análisis tradicional al actuar como especies diferenciales de las distintas intensidades de manejo (San Martín et al., 2014). Cómo las turberas más inter- venidas, presentan menos especies y una dominancia considerable de Sphagnum magellanicum, ellas son las más favorables para una explotación del musgo, que se presenta limpio y no contaminado con otras especies (Figura 5). No obstante, como estas formaciones vegetales representan humedales que actúan como grandes reservorios de agua que regulan la escorrentía y la evaporación (Camacho y Ruiz, 2012), la explotación del musgo debe hacerse de un modo racional, asegurando su regeneración, para no afectar el ciclo hídrico.

\section{CONCLUSIONES}

Aunque las tres turberas investigadas pertenecen a la misma asociación vegetal, se encontraron importantes diferencias en su composición florística, las que al ser analizada con estadística multivariable de clasificación y ordenación es posible identificar una segregación en dos gradientes ecológicos, asimilables con anegamiento y contenido de materia orgánica en el sustrato,
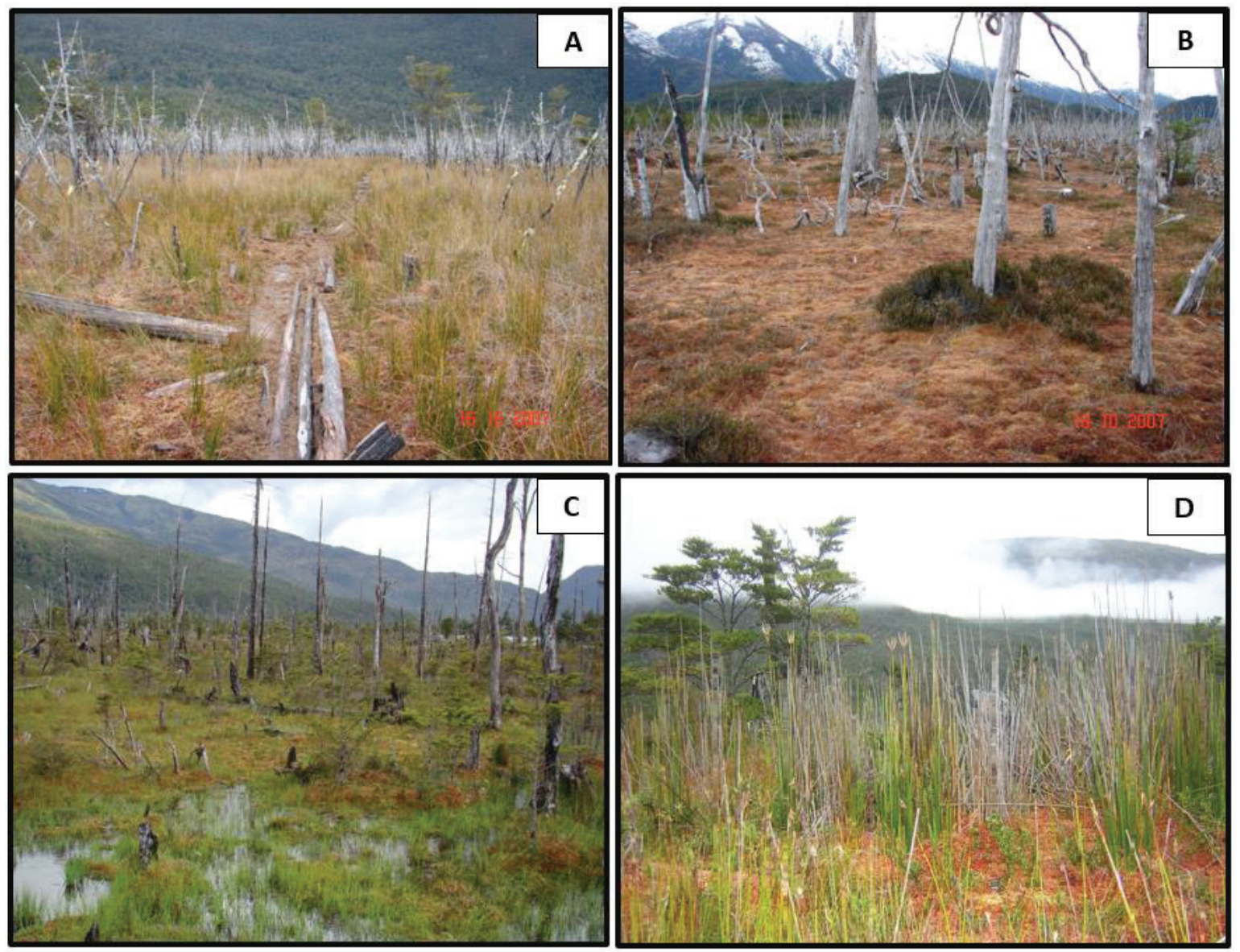

Figura 5. Aspecto de las turberas esfagnosas trabajadas. $A=$ Predio de José Iñiguez, $B=$ Predio Guelet, $C=$ Predio Guzmán, $\mathrm{D}=$ Detalle de la presencia de Marsippospermum grandiflorum (Primer plano) en la turbera del Predio Iñiguez.

Figure 5. Appearance of worked peat bogs: $A=$ José Iñiguez farm, $B=$ Guelet farm, $C=$ Guzmán farm, $D=$ Detail of the presence of Marsippospermum grandiflorum (Foreground) in the Iñiguez farm bog. 
que explican la mayor variación. Las diferencias florísticas encontradas entre los tres predios son asimilables a las diferencias de manejo entre ellos, lo que permitió estimar diferentes intensidades de intervención en cada uno. Las especies diferenciales de las tres turberas aparecen como indicadoras de las distintas condiciones alcanzadas con los diferentes manejos lo que es confirmado por el análisis de especies indicadoras con altos valores de probabilidad, de maneras que ellas podrían servir como indicadoras de las condiciones ambientales en la turbera, que serían diferentes según el manejo.

\section{AGRADECIMIENTOS}

Investigación financiada por el Proyecto FIA-PIC-2044-1 "Evaluación cuantitativa y cualitativa del musgo Sphagnum sp., como forma de conocer su uso, manejo y protección en la Comuna de Tortel, XI Región".

\section{REFERENCIAS}

Álvarez, M., San Martín, C., Novoa, C., Toledo, G., Ramírez, C., 2010. Diversidad florística, vegetacional y de hábitats en el Archipiélago de Los Chonos (Región de Aisén, Chile). Anales del Instituto de la Patagonia (Chile) 38(1), 35-56. http://dx.doi.org/10.4067/S0718686X2010000100002

Álvarez, M., Möseler, B.M., San Martín, C., 2013. Tendency and distribution indices for ordinal variables applied to Ellenberg indicator values. Gayana Botánica 70(2), 247-255. http://dx.doi.org/10.4067/S071766432013000200006

Amigo, J., Ramírez, C., 1998. A bioclimatic classification of Chile: woodland communities in the temperate zone. Plant Ecology 136(1), 9-26. https://doi. org/10.1023/A:1009714201917

Amigo, J., San Martín, C., Ramírez, C., Álvarez, M., 2017. Nomenclatural revision and syntaxonomical proposal for wetland peat vegetation in the Valdivian-Magellanian region. Lazaroa 38(2), 165-187. http://dx.doi. org/10.5209/LAZA.56343

Badii, M., Landeros, J., Cerna, E., 2008. Patrones de asociación de especies y sustentabilidad. Daena: International Journal of Good Conscience 3(1), 632-660.

Braun-Blanquet, J., 1979. Fitosociología, bases para el estudio de las comunidades vegetales. H. Blume Ediciones, Madrid, España.

Camacho, V., Ruiz, L., 2012. Marco conceptual y clasificación de los servicios ecosistémicos. Revista Bio Ciencias 1(4), 3-15.

Clarke, K.R., Ainsworth, M., 1993. A method of linking multivariate community structure to environmental variables. Marine Ecology Progress Series 92(3), 205-219. https://doi.org/10.3354/meps092205

Dec, D., Zúñiga, F., Thiers, O., Paulino, L., Valle, S., Villagra, V., Tadich, I., Horn, R., Dörner, J., 2017. Water and temperature dynamics of Aquands unter different uses in southern Chile. Journal of Soil Science and Plant Nutri- tion 17(1), 141-154. http://dx.doi.org/10.4067/S071895162017005000011

Dengler, J., Chytry, M., Ewald, J., 2008. Phytosociology, in: Jörgensen, S.E., Fath, B.D. (Eds.), Encyclopedia of Ecology. Elsevier, Oxford, England, pp. 2767-2779.

Díaz, M., Larraín, J., Zegers, G., Tapia, C., 2008. Caracterización florística e hidrológica de turberas de la Isla Grande de Chiloé, Chile. Revista Chilena de Historia Natural 81(4), 455-468. http://dx.doi.org/10.4067/S0716078X2008000400002

Diekmann, M., 2003. Species indicator values as an important tool in applied plant ecology - a review. Basic and Applied Ecology 4(6), 493-506. https://doi. org/10.1078/1439-1791-00185

Dierschke, H., 1994. Pflanzensoziologie-Grundlagen und Methoden. Eugen Ulmer, Stuttgart.

Dufrene, M., Legendre, P., 1997. Species assemblages and indicator species: the need for a flexible asymmetrical approach. Ecological Monographs 67(3), 345-366. https:// doi.org/10.1890/0012-9615(1997)067[0345:SAAIST] 2.0.CO;2

Frey, W., Lösch, R., 2010. Geobotanik Pflanze und Vegetation in Raum und Zeit. Spektrum Akademischer Verlag, Heidelberg.

Hajek, E., Di Castri, F., 1975. Bioclimatografía de Chile. Editorial Universidad Católica de Chile, Santiago.

Knapp, R., 1984. Considerations on quantitative parameters and qualitative attibutes in vegetation analysis and in phytosociological relevés, in: Knapp, R. (Ed.), Sampling methods and taxon analysis in vegetation science. Dr. W, Junk Publishers, La Haya, pp. 77-119.

Legendre, P., Legendre, L., 1998. Numerical ecology. Elsevier, Amsterdam. Holland.

Luebert, F., Pliscoff, P., 2006. Sinopsis bioclimática y vegetacional de Chile. Editorial Universitaria, Santiago, Chile.

McCune, B., Grace, J.B., 2002. Analysis of ecological communities. MJM Software, Glenden Beach, Oregon, USA.

Mueller-Dombois, D., Ellenberg, H., 1974. Aims and methods of vegetation ecology. Wiley \& Sons, New York, USA.

Oberdorfer, E., 1960. Pflanzensoziologischen Studien in Chile - Ein Vergleich mit Europa. Flora et Vegetatio Mundi 2, 1-208.

Pisano, E., 1983. The Magellanic tundra complex, in: Gore, A.J.P. (Ed.), Ecosystems of the World, 4. Mires: Swamp, Biog, Fen and Moor, B Regional Studies. Elsevier Science Publishers, Amsterdan, Holland, pp. 289-323.

Promis, A., 2010. Humedales anegadizos de la Patagonia chilena. Ambiente Forestal 5, 6-16.

Ramírez, C., Correa, M., Figueroa, H., San Martín, J., 1985. Variación del hábito y hábitat de Nothofagus antarctica en el centro-sur de Chile. Bosque 6(2), 55-73.

Ramírez, C., San Martín, C., Uribe, F., Mac Donald, R., 1993. La vegetación nativa de los suelos de ñadi valdivianos (Chile). Agricultura Técnica (Chile) 53(1), 55-74.

Ramírez, C., Sandoval, V., San Martín, C., Álvarez, M., Pérez, Y., Novoa, C., 2012. El paisaje rural antropogénico de Aisén, Chile: Estructura y dinámica de la vegetación. Gayana Botánica 69(2), 219-231. http://dx.doi.org/10.4067/ S0717-66432012000200001

Ramírez, C., Fariña, J.M., Camaño, A., San Martín, C., Pérez, Y., Solís, J.L., Valdivia, O., 2018. The case of the Itata estuary 
(Bio-Bio Region-Chile) plant formations: anthropogenic interference or natural disturbance-induced diversity enrichment? Mediterranean Botany 39(1), 17-34. https://doi.org/10.5209/MBOT.59427

Rodríguez, R., Marticorena, A., Teneb, E., 2008. Plantas vasculares de los ríos Baker y Pascua, Región de Aisén, Chile. Gayana Botánica 65(1), 39-70. http://dx.doi. org/10.4067/S0717-66432008000100006

Roig, F., Dollenz, O., Méndez, E., 1983. Tundra y bosque magallánico. Su dinamismo en el Sur de Chile. Serie Científica de Mendoza 30, 5-10.

San Martín, C., Ramírez, C., Figueroa, H., 1999. Análisis multivariable de la vegetación de un complejo de turberas en Cordillera Pelada (Valdivia, Chile). Lazaroa 20, 95106.

San Martín, C., Ramírez, C., Álvarez, M., 2004. Estudio de la vegetación de "mallines" y "campañas" en la Cordillera Pelada (Valdivia, Chile). Revista Geográfica de Valparaíso $35,261-273$.

San Martín, C., Sandoval, V., Álvarez, M., Vidal, O., Pérez, Y., Solís, J.L., 2014. Comparación de etapas de degradación vegetacional con manejo pecuario utilizando valores bioindicadores de Ellenberg en la Patagonia Chilena. Bosque 35(2), 141-154. https://doi.org/10.4067/ S0717-92002014000200002

Teneb, E., Gómez, P., González, M., 2008. Observaciones sobre la flora y vegetación de dos turberas en la Región de Aisén, Patagonia chilena. Gayana Botánica 65(2), 229-232. http://dx.doi.org/10.4067/S071766432008000200009

Thiers, O., Gerding, V., Lara, A., Echeverría, C., 2007. Variación de la napa freática en un suelo ñadi bajo diferentes tipos vegetacionales, X Región, Chile., en: Gonda, H., Davel, M., Loguercio, G., Picco, O.A. (Eds.), Libro de Actas de Eco Reuniones. Primera reunión sobre forestación en la Patagonia EcoForestar 2007. Centro de Investigación y Extensión Forestal Andino Patagónica (CIEFAP), Argentina, pp. 259-266.

Walter, H., 1997. Zonas de vegetación y clima. Ediciones Omega, Barcelona, España.

Wikum, D., Shanholtzer, G.F., 1978. Application of the BraunBlanquet cover-abundance scale for vegetation analysis in land development studies. Environmental Management 2(4), 323-329. https://doi.org/10.1007/ BF01866672

Zegers, G., Larraín, J., Díaz, M.F., Armesto, J., 2006. Impacto ecológico y social de la explotación de pomponales y turberas de Sphagnum en la Isla Grande de Chiloé. Revista Ambiente y Desarrollo de CIPMA 22(1), 28-34.

Zuloaga, F., Morrone, O., Belgrano, M., 2008. Catálogo de las plantas vasculares del cono sur. Missouri Botanical Garden Press, Missouri. 
